\title{
Stromal ColXa1 expression correlates with tumor-infiltrating lymphocytes and predicts adjuvant therapy outcome in ER-positive/ HER2-positive breast cancer
}

Chaohui Lisa Zhao ${ }^{1}$, Kamaljeet Singh², Alexander S. Brodsky', Shaolei Lu', Theresa A. Graves³, Mary Anne Fenton ${ }^{4}$, Dongfang Yang ${ }^{1}$, Ashlee Sturtevant ${ }^{1}$, Murray B. Resnick ${ }^{1}$ and Yihong Wang ${ }^{1 *}$ (i)

\begin{abstract}
Background: The breast cancer microenvironment contributes to tumor progression and response to chemotherapy. Previously, we reported that increased stromal Type X collagen a1 (ColXa1) and low TILs correlated with poor pathologic response to neoadjuvant therapy in estrogen receptor and HER2-positive (ER+/HER2+) breast cancer. Here, we investigate the relationship of ColXa1 and long-term outcome of ER+/HER2+ breast cancer patients in an adjuvant setting.

Methods: A total of 164 cases with at least 5-year follow-up were included. Immunohistochemistry for ColXa1 was performed on whole tumor sections. Associations between ColXa1expression, clinical pathological features, and outcomes were analyzed.

Results: ColXa1 expression was directly proportional to the amount of tumor associated stroma $(p=0.024)$ and inversely proportional to TILs. Increased ColXa1 was significantly associated with shorter disease free survival and overall survival by univariate analysis. In multivariate analysis, OS was lower in ColXa1 expressing $(\mathrm{HR}=2.1 ; 95 \% \mathrm{Cl}=$ 1.2-3.9) tumors of older patients ( $>=58$ years) $(\mathrm{HR}=5.3 ; 95 \% \mathrm{Cl}=1.7-17)$ with higher stage $(\mathrm{HR}=2.6 ; 95 \% \mathrm{Cl}=1.3-$ 5.2). Similarly, DFS was lower in ColXa1 expressing $(H R=1.8 ; 95 \% \mathrm{Cl}=1.6-5.7)$ tumors of older patients $(H R=3.2$; $95 \% \mathrm{Cl}=1.3-7.8)$ with higher stage $(\mathrm{HR}=2.7 ; 95 \% \mathrm{Cl}=1.6-5.7)$ and low TILs. In low PR+ tumors, higher ColXa1 expression was associated with poorer prognosis.

Conclusion: ColXa1 expression is associated with poor disease free survival and overall survival in ER+/HER2+ breast cancer. This study provides further support for the prognostic utility of ColXa1 as a breast cancer associated stromal factor that predicts response to chemotherapy.
\end{abstract}

Keywords: Collagen, Tumor infiltrating lymphocytes, Tumor microenvironment, Breast cancer, Adjuvant chemotherapy

\section{Background}

Breast cancer is the second leading cause of cancerrelated death in women. HER2 targeting therapies, such as trastuzumab and pertuzumab, prolong survival in breast cancer patients [1, 2]. HER2-positive breast cancers constitute a heterogeneous disease at the molecular

\footnotetext{
* Correspondence: yihong_wang@brown.edu

${ }^{1}$ Department of Pathology and Laboratory Medicine, Rhode Island Hospital and Lifespan Medical Center, Warren Alpert Medical School of Brown University, 593 Eddy St, APC 12, Providence, RI 02903, USA

Full list of author information is available at the end of the article
}

level. The repertoire of somatic genetic alterations in these tumors varies according to estrogen receptor (ER) status and correlates with "intrinsic" subtype [3-6]. Early studies focusing on breast cancer molecular subtypes showed that Luminal-B subtype has poorer outcome than luminal-A subtype. In fact, the overall survival in untreated luminal-B subtype is similar to basal-like and HER2-positive tumors, which are widely recognized as high risk [3]. The treatment outcome for patients with ER+/HER2+ cancer, sometimes referred to as Luminal HER2 type, is variable [7-9]. Therefore, additional

(c) The Author(s). 2019 Open Access This article is distributed under the terms of the Creative Commons Attribution 4.0 International License (http://creativecommons.org/licenses/by/4.0/), which permits unrestricted use, distribution, and reproduction in any medium, provided you give appropriate credit to the original author(s) and the source, provide a link to the Creative Commons license, and indicate if changes were made. The Creative Commons Public Domain Dedication waiver (http://creativecommons.org/publicdomain/zero/1.0/) applies to the data made available in this article, unless otherwise stated. 
biomarkers are needed for risk stratification and treatment response prediction. We previously reported that increased stromal Type X collagen $\alpha 1$ (ColX $\alpha 1)$ expression in ER+/HER2+ tumors is associated with poor response to neoadjuvant therapy. Our findings provided initial evidence that a specific stromal collagen subtype in the breast tumor microenvironment predicts therapy response [10].

Tumor associated stroma is composed of a matrix of fibronectin, matrix metalloproteinases, collagens and other connective tissue proteins which undergo constant remodeling [11]. Tumor-stromal interactions play a key role in tumorigenesis and metastasis influencing the prognosis of several human malignancies [12-14]. Some studies have reported that tumor microenvironment components like tumor infiltrating lymphocytes (TILs) and tumor-associated stroma predict response to therapy and breast cancer progression [15]. In this study we aimed to evaluate the prognostic and predictive value of ColX $\alpha 1$ expression in the tumor stroma of ER+/HER2+ breast tumors in the adjuvant setting.

\section{Methods}

\section{Patients and tissue samples}

The study was approved by the ethics committees of Lifespan Medical Center (467617-9) and Women Infants Hospital (797108-3). The need for consent was waived by the IRB.

A retrospective search was performed in the cancer registry database for breast cancer patients who received adjuvant therapy at Lifespan Medical Center and Women and Infant Hospital in Rhode Island between 2007 and 2013. We identified 164 ER+/HER2+ cases who received adjuvant chemotherapy and HER2targeted therapy. All original tumor slides were reviewed and histological features were recorded. Immunohistochemistry for ER, PR, and HER2 expression were classified according to the CAP/ASCO guidelines $[16,17]$. ER and PR positive cases were further classified as lowpositive $(1-10 \%)$ and positive $(>10 \%)$.

\section{Immunohistochemistry and ColXa1 expression scoring}

For all cases, 4- $\mu$ m-thick tissue sections were cut from formalin-fixed paraffin-embedded tumor tissue and subjected to immunohistochemical staining according to the manufacturers' protocol as previous described [10]. Anti-ColX $\alpha 1$ (1:50, eBioscience/Affymetrix, Clone X53), ER (1:50, DAKO, clone 1D5), PR (1:400, DAKO, clone 1A6), HER2 (DAKO HercepTestTM), and monoclonal mouse anti-human Ki-67 (clone MIB1, Ready-to-use, Dako) were used for immunohistochemistry. ColX $\alpha 1$ was scored as previously described [10]. Briefly, no staining was scored as 0 ; weak staining as $1+;<10 \%$ of stroma tissue with intense staining present as $2+;>10 \%$ of stroma tissue with intense staining as $3+$ (Fig. 1). ColX $\alpha 1$ immunohistochemical stain was further grouped into low (scores 0-1) and high expression (scores 2-3) categories. Ki-67 was scored as percentage of tumor cells with nuclear staining by two pathologists (YW and KS) independently on fresh cut slides. The Ki-67 score was calculated for each patient by averaging the two pathologists readings.

\section{Tumor-associated stroma and TILs analysis}

We morphologically evaluated the amount of intratumoral stroma and TILs on tumor samples. The stromal evaluation protocol has been previously published [10]. Briefly, the amount of tumor-associated stroma was scored as 0 to 2: 0 for absent or minimal stroma $(<10 \%)$, 1 for mild to moderate amount of stroma (10-40\%) and 2 for abundant stroma (>40\%). The TILs were evaluated based on criteria published by Denkert et al. [15]. Briefly, iTILs are defined as lymphocytes in direct contact with the tumor cells, whereas sTILs are defined as lymphocytes in the surrounding stroma with the percent of the tumor or stromal volume comprised of infiltrating lymphocytes. The results were evaluated in increments of 10 (0-1\% was scored as 0 , with all other estimates rounded up to the next highest decile [for e.g. 11-20\% was scored as 20]). sTILs and iTILs were added to calculate TILs. The trends were similar for each lymphocyte fraction (data not shown). We chose to analyze sTILs as they are considered as the most consistent metric (as recommended by the International TILs Working Group) [18]. While analyzing survival, the sTILs data was divided into three groups. Group 1: Rare TILs in the stroma (0-10\%). Group 2: low density TILs (11-59\%) and Group 3: high density TILs (> = 60\%).

\section{Gene expression and pathway analysis}

TCGA RNA-seq data for breast invasive carcinoma was downloaded from the Firehose Broad GDAC [19]. TCGA clinical data was downloaded from the TCGA data archive in September 2015 (http://cancergenome. nih.gov/).

\section{Statistical analysis}

Fisher Exact test and Chi-square test were used when appropriate. T-test or analysis of variance (ANOVA) was used to compare continuous variables. For time-to-event measures, the Kaplan-Meier method was used to estimate the empirical survival and log-rank estimates were used. Relationships between variables were assessed using Pearson and Spearman correlation analysis as noted. Multivariate analysis was performed using the Cox proportional hazards model. Statistical analysis was performed utilizing SPSS v25 (SPSS, Chicago, IL, USA). 


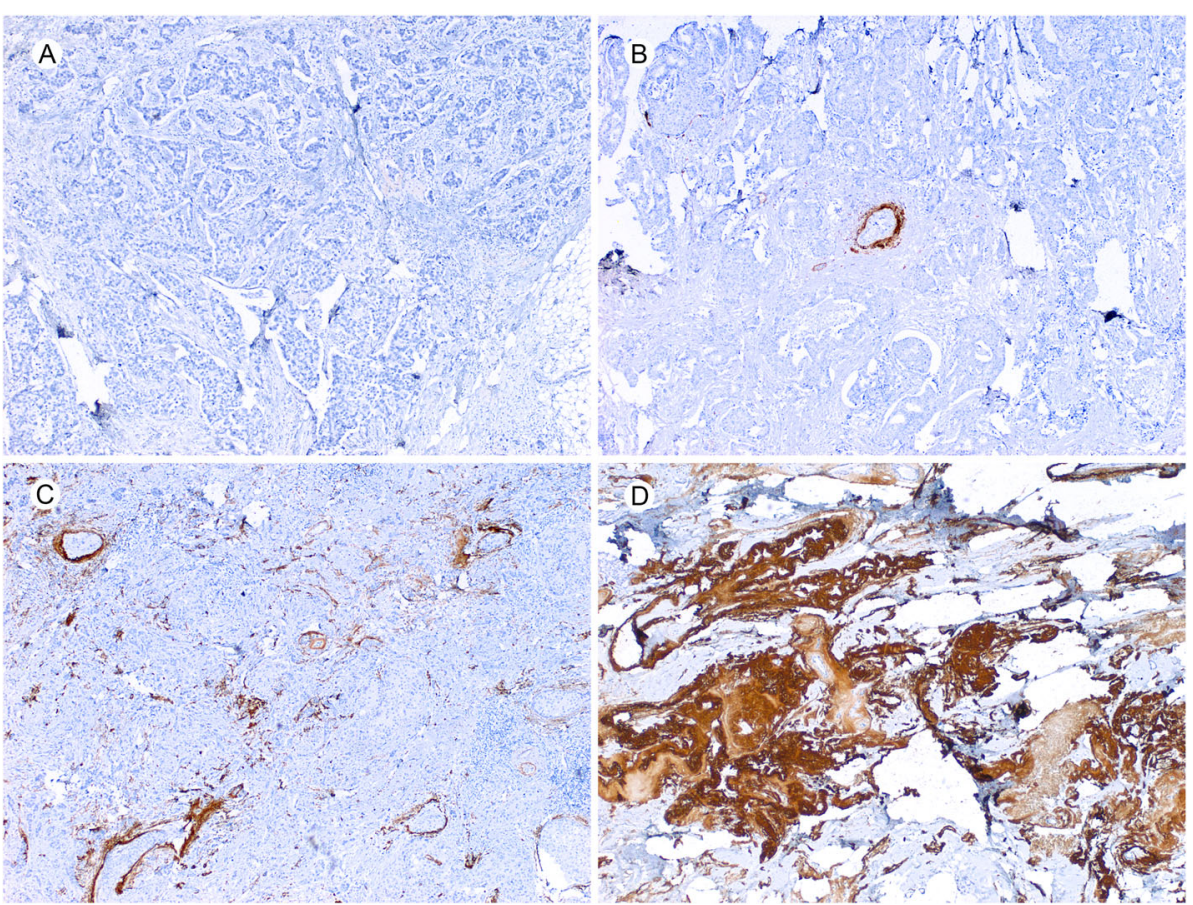

Fig. 1 Photomicrographs of stromal ColXa1 immunohistochemical staining (40X). a No expression (score 0). b Weak expression (score 1). c Moderate expression (score 2). d Strong expression (score 3) (× 200)

A $P$-value $<0.05$ was considered statistically significant. All $P$-values reported are two-sided.

\section{Results}

\section{Patient characteristics and clinical pathologic features}

A total of 164 ER+/HER2+ breast cancer patients who received adjuvant therapy and with at least a five year follow up were included in the study. All data generated or analyzed during this study were de-identified and included in this published article (Additional file 1: Table S1). The clinical and pathologic data of the patients are summarized in Table 1. Mean age was 60 years (median $=58$; range $=25-99)$. There were $142(87 \%)$ ductal, $9(5 \%)$ lobular and $13(8 \%)$ mixed ductal and lobular carcinomas. Out of 164 cases, 120 (73\%) underwent lymph node sampling including 86 (72\%) axillary dissections and 34 (28\%) sentinel lymph node biopsies. There were $66(40 \%)$ grade 2, 95 (58\%) grade 3 and 3 (2\%) grade 1 tumors. Lymphovascular invasion (LVI) was identified in 95/130 cases (73\%) and lymph node metastasis were present in 56/120 (47\%) cases. As expected for the ER+/HER2+ group, there were 87 (53\%) stage II, 52 (31\%) stage I and 22 (13\%) stage III cases. Out of 164 cases, 86 (52.4\%) had only rare TILs, 57 (34.8\%) had low density TILs and 21 (12.8\%) tumors showed high density TILs. The Ki-67 IHC was performed on 144 cases, of which 133 (95\%) tumors had a Ki-67 index $>=20 \%$ (median $=40$, range $=20-90$ ) consistent with a luminal B intrinsic subtype. Seven (5\%) tumors with
Ki-67 index estimated $<20 \%$ were positive for ColX $\alpha 1$. The mean Ki-67 index for grade 2 tumors $(34.8$; 95\% CI = 29.8-39.9) was significantly lower than grade 3 tumors (44.9; 95\% CI $=40.5-49.3$ ).

\section{Correlation of ColXa1 with other factors and TILs}

Overall ColX $\alpha 1$ positivity was present in 143/164 (87\%) cases. ColX $\alpha 1$ expression was directly proportional to the amount of stroma in the tumor $(p=0.024)$ and correlated with the presence of LVI $(p=0.008)$. There was a significant inverse relationship between TILs and ColX $\alpha 1$ expression by immunohistochemistry $(\mathrm{r}=-0.47$; $p<0.001$, Table 2). A significantly higher proportion of tumors with rare and low density TILs were ColX $\alpha 1$ positive when compared to the high density TILs group $(P<0.001$, Table 1$)$. ColX $\alpha 1$ expression was present in $15 / 22(68.2 \%)$ cases with rare TILs. Similarly, 82/113 (72.6\%) of tumors with low density TILs were ColX $\alpha 1$ positive. Conversely, only 6/29 (20.7\%) high density TILs cases had ColX $\alpha 1$ expression.

\section{Correlation of ColXa1 and TILs and tumor associated immune cells}

In the TCGA cohort, a similar trend of inverse relationship between the tumor immune microenvironment and ColX $\alpha 1$ expression by RNA-seq was noted (Table 2). There was an inverse relationship between ColX $\alpha 1$ and lymphocytes $(\mathrm{r}=-0.33 ; p=0.001)$, Naïve B cells $(\mathrm{r}=-$ 
Table 1 Clinicopathological characteristics of cohort. Fisher's exact test and Chi-square test (as appropriate) were used to generate $P$-values

\begin{tabular}{|c|c|c|c|}
\hline Characteristic & No. & ColXa1 Positive N (\%) & P-value \\
\hline No. of patients & 164 & $62.8 \%$ & \\
\hline Age (year) & 164 & & \\
\hline$<65$ & 99 & $57(57.5 \%)$ & 0.10 \\
\hline$\geq 65$ & 65 & $46(71 \%)$ & \\
\hline \multicolumn{4}{|l|}{ Age (year) } \\
\hline$<58$ & & $61 \%$ & 0.75 \\
\hline$\geq 58$ & & $64 \%$ & \\
\hline Laterality & 164 & & \\
\hline Left & 94 & $54(57.3 \%)$ & 0.52 \\
\hline Right & 70 & $30(42.7 \%)$ & \\
\hline Foci & 164 & & \\
\hline Solitary & 146 & $61.6 \%$ & 0.45 \\
\hline Multifocal & 18 & $72.2 \%$ & \\
\hline LVI & 130 & & \\
\hline Pos & 97 & $62(63.9 \%)$ & 0.005 \\
\hline Neg & 33 & $21(63.6 \%)$ & \\
\hline Lymph node status & 120 & & 0.21 \\
\hline NO & 64 & $30(46.9 \%)$ & \\
\hline N1 & 42 & $26(61.9 \%)$ & \\
\hline $\mathrm{N} 2$ & 8 & $4(50.0 \%)$ & \\
\hline N3 & 6 & $5(83.3 \%)$ & \\
\hline Clinical stage & 164 & & \\
\hline । & 52 & $61.5 \%$ & 0.34 \\
\hline$\|$ & 87 & $60.9 \%$ & \\
\hline III & 22 & $77.3 \%$ & \\
\hline IV & 3 & $33.3 \%$ & \\
\hline ColXa1 & 164 & & \\
\hline 0 & 21 & & - \\
\hline 1 & 40 & & \\
\hline 2 & 47 & & \\
\hline 3 & 56 & & \\
\hline Stroma Content & 164 & & \\
\hline 0 & 30 & $15(50 \%)$ & 0.024 \\
\hline 1 & 95 & $57(60 \%)$ & \\
\hline 2 & 39 & 31 (79.5\%) & \\
\hline TILS & 164 & & \\
\hline 1 (Rare) & 22 & $15(68.2 \%)$ & $<0.001$ \\
\hline 2 (Low density) & 113 & $82(72.6 \%)$ & \\
\hline 3 (High density) & 29 & $6(20.7 \%)$ & \\
\hline
\end{tabular}

$0.22 ; p=0.02), \mathrm{CD} 8 \mathrm{~T}$ cells $(\mathrm{r}=-0.24 ; \mathrm{p}=0.02)$ and monocytes $(\mathrm{r}=-0.26 ; p=0.008)$. A positive correlation was present between ColX $\alpha 1$ RNA-seq and macrophages
Table 2 Spearman and Pearson Correlations of ColXa1 with Tumor Immune Environment

\begin{tabular}{lll}
\hline Characteristic & Pearson with ColXa1 & P-value \\
\hline RIH dataset & ColXa1 IHC & \\
TILs & -0.47 & $<0.001$ \\
Age & 0.099 & 0.21 \\
TCGA (ER/HER2 only, N = 102) Spearman with ColXa1 RNA-seq \\
Naïve B Cells & -0.29 & 0.003 \\
CD 8T Cells & -0.36 & $<0.001$ \\
Monocytes & -0.28 & 0.005 \\
Lymphocytes & -0.41 & $<0.001$ \\
Macrophages & 0.40 & $<0.001$ \\
TIL Percentage & -0.01 & 0.32 \\
\hline
\end{tabular}

$(\mathrm{r}=0.35 ; p<0.001)$. The TCGA cohort, like our group, showed an inverse relationship between TILs and ColX $\alpha 1$, however the $p$-value did not reach statistical significance $(\mathrm{r}=-0.15 ; p=0.1)$.

\section{ColXa1, TILs and survival}

A statistically significant difference in breast cancer disease free survival (DFS) $(\mathrm{HR}=1.8 ; 95 \% \mathrm{CI}, 1.6$ to 5.7 ) and overall survival (OS) was found between high and low ColX $\alpha 1$ tumors ( $\mathrm{HR}=2.1 ; 95 \% \mathrm{CI}, 1.2$ to 3.9$)$ by cox proportional hazards analysis (Table 3 ). Similar survival trends were noted in the TCGA cohort (Fig. 2). In a multivariate cox proportional hazards analysis, OS was lower in ColX $\alpha 1$ expressing $(\mathrm{HR}=2.1,95 \% \mathrm{CI}, 1.2$ to 3.9) older patients ( $\mathrm{HR}=5.3,95 \% \mathrm{CI}, 1.7$ to 17$)$ with higher stage ( $\mathrm{HR}=2.6,95 \% \mathrm{CI}, 1.3$ to 5.2$)$ and low TILs $(\mathrm{HR}=0.96$; 95\% CI, 0.9-1.0). Similarly, DFS was lower in ColXo1 expressing ( $\mathrm{HR}=1.8,95 \% \mathrm{CI}, 1.6$ to 5.7$)$ older patients $(\mathrm{HR}=3.2,95 \% \mathrm{CI}, 1.3$ to 7.8$)$ with higher stage $(\mathrm{HR}=2.7,95 \% \mathrm{CI}, 1.6$ to 5.7$)$ and low TILs (HR $=0.98$; 95\% CI, 0.94-1.0) (Table 4). We noted a relationship between PR status, ColX $\alpha 1$ and OS in our cohort. In low PR group of 48 (29\%) tumors, OS was significantly lower in ColX $\alpha 1$ high tumors. ColX $\alpha 1$ expression was associated with poor outcome in low PR tumors but not high PR tumors by stratification Kaplan-Meier analysis (Fig. 3).

\section{Discussion}

The HER2+ breast cancers are a heterogeneous group with variable tumor aggressiveness and response to therapy $[20,21]$. HER2+ tumors can be further classified according to the hormone receptor status. Luminal-B subtype is defined as ER+ tumor with increased tumor cell proliferation which is usually assessed by KI-67 [22]. The ER+/HER2 + tumors are also classified as luminal-B subtypes, and sometimes referred to as Luminal HER2 subtype [6]. In our cohort of Luminal B HER2 subtype, most tumors (95\%) had high KI-67 expression (> = 20\%). 
Table 3 Cox Proportional Hazards Univariate Analysis ( $N=158$ unless otherwise noted). 95\% confidence levels are indicated in the parentheses for the Hazard Ratios (HR)

\begin{tabular}{|c|c|c|c|c|}
\hline Characteristic & HR OS & POS & HR PFS & P PFS \\
\hline Clinical Stage & $2.0(1.1-3.4)$ & 0.02 & $2.1(1.4-3.3)$ & $<0.001$ \\
\hline Grade & 1 & 1.0 & $1.1(0.5-2.3)$ & 0.77 \\
\hline Age & $1.08(1.05-1.1)$ & $<0.001$ & $1.05(1.0-1.1)$ & $<0.001$ \\
\hline Lymph Node Status $(N=120)$ & $2.5(1.4-4.5)$ & 0.002 & $2.7(1.6-4.4)$ & $<0.001$ \\
\hline PR status & $1.1(0.4-3.1)$ & 0.83 & $1.1(0.5-2.7)$ & 0.76 \\
\hline Stroma & $1.1(0.6-2.3)$ & 0.73 & $1.1(0.6-2.0)$ & 0.72 \\
\hline TILS & $0.96(0.92-1.0)$ & 0.027 & $0.97(0.9-1.0)$ & 0.025 \\
\hline ColXa1 & $2.3(1.3-4.1)$ & 0.006 & $1.8(1.2-2.8)$ & 0.008 \\
\hline
\end{tabular}

Although some studies report better outcome in Luminal HER2 breast cancer when compared to ERnegative HER2-positive breast cancer [20-23], others have found no significant difference in long term survival [24-26]. Metastatic sites and recurrence patterns of HER2 + tumors are ER dependent. Bone is the most common site of distant metastasis for Luminal HER2 subtype, which is similar to Luminal A cancers, whereas ER-negative HER2-positive cancers have the higher rates of locoregional recurrence and these tend to initially metastasize to visceral organs such as lung [23, 26, 27]. In ER+/HER2+ breast cancer, HER2 overexpression predicts poor response to hormone therapy [28, 29]. Cross talk between HER2 and ER results in persistent activation of the hormone receptor downstream signaling, even in the presence of hormone treatment [30].

The tumor microenvironment consists of non-cellular elements such as collagens, glycosaminoglycans, proteoglycans and hyaluronic acid. Collagens are major components of the extracellular matrix of breast tumors. Collagen expression patterns have been linked with aggressive tumor behavior and drug resistance [31]. Together with hyaluronic acid, collagen accumulation is also associated with high tumoral interstitial pressure, vascular collapse and drug resistance [32, 33]. Myriad collagen genes are critical in tissue development and physiological function, as evidenced by the range of collagenopathies [34]. Nevertheless, the mechanistic role of collagen subtypes in cancer progression has been largely overlooked, although they frequently emerge as components of tumor stromal expression signatures [35, 36]. A variety of collagen subtypes are highly expressed in breast tumors contributing to its dense structure. The alignment of collagen fibers has been proposed to indicate progression in breast tumors [37, 38]. ColX $\alpha 1$ land its hexagonal network that plays a role in tissue stiffness has been associated with chemoresistance and breast cancer progression [39]. Collagen

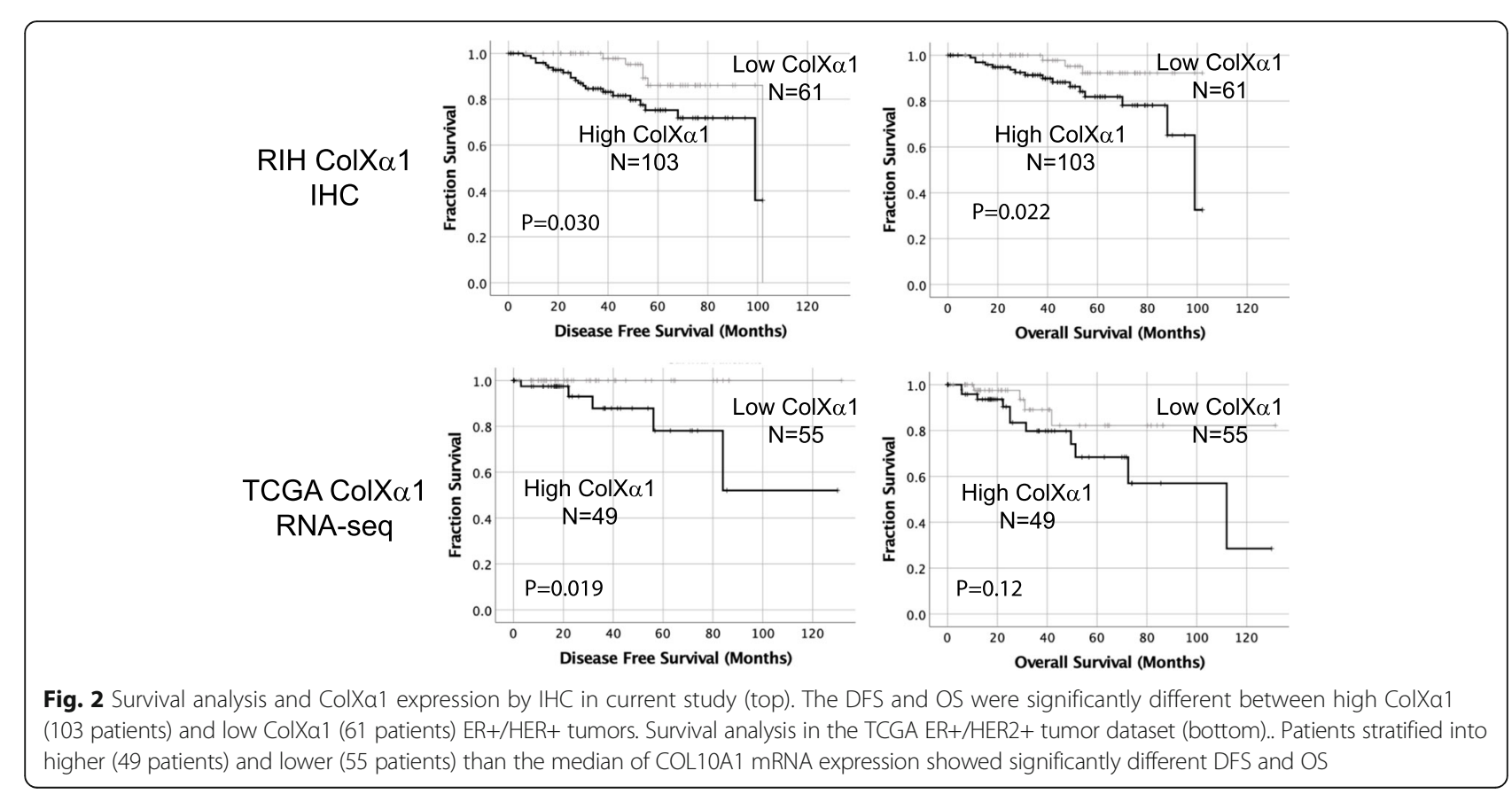


Table 4 Multivariate analysis of variables with $p<0.05$ and significant $\mathrm{HR}$ as univariate for overall and progression free survival ( $N=158) .95 \%$ confidence levels are indicated in the parentheses for the Hazard Ratios (HR)

\begin{tabular}{lllll}
\hline Factor & HR OS & P OS & HR PFS & P PFS \\
\hline Age $(>$ 58) & $5.3(1.7-17.0)$ & 0.005 & $3.2(1.3-7.8)$ & 0.01 \\
ColXa1 & $2.1(1.2-3.9)$ & 0.01 & $1.8(1.6-5.7)$ & 0.01 \\
Clinical Stage & $2.6(1.3-5.2)$ & 0.008 & $2.7(1.6-4.7)$ & $<0.001$ \\
\hline
\end{tabular}

type $\mathrm{X}$ mRNA expression is up-regulated in a variety of human malignancies when compared to normal tissue, including breast tumors [40]. In colorectal cancer, Huang and colleagues demonstrated that ColX $\alpha 1$ expression was significantly higher in the tumor stroma compared with normal tissues. ColX $\alpha 1$ enhanced proliferation, migration, invasion of colon cancer cells and knockdown of ColX $\alpha 1$ inhibited tumorigenesis in vivo [41]. Similar to the findings in the colorectal tissue, we reported that ColX $\alpha 1$ was not expressed in normal breast tissue [10]. Interestingly ColX $\alpha 1$ is present in a periductal location surrounding ductal carcinoma in situ, and within the tumor associated stroma in select invasive breast carcinomas, particularly in ER+/HER2+ cancers [42].

We had earlier identified that presence of ColX $\alpha 1$, determined by gene expression analysis and immunohistochemistry, predicted neoadjuvant therapy response in ER+/HER2+ tumors. In the current study, we demonstrated that ColX $\alpha 1$ expression predicts long term outcome in ER+/HER2+ tumors in the adjuvant setting as well. In our cohort most of the patients were treated before the results of the Z-11 trial were published and axillary dissection was performed in a significant number of cases. Consistent with other studies [3, 7-9], ER+/ HER+ tumors exhibited aggressive features; three quarters of the cases had LVI, and half of the cases had lymph node metastasis.

We found an inverse relationship between TILs and ColX $\alpha 1$ expression, and that low TILs and ColX $\alpha 1$ both contribute to the poor treatment response and prognosis. We did not found differences in TIL or ColX $\alpha 1$ expression with age. The relationship of ColXa1 expression and imaging findings, like mammographic density, can be investigated in future studies.

In addition, ER and PR positivity is defined by $>1 \%$ of nuclear staining of the tumor cells. In practice, a large amount of information is lost when one labels a tumor as a ER or PR-positive, because a tumor in which $10 \%$ of cells exhibit weak ER or PR staining is biologically different from one that demonstrates strong intensity staining in about $90 \%$ of cells. Although the vast majority of hormone receptor positive tumors show strong immunoreactivity, approximately $20 \%$ of tumors exhibit variable ER/PR expression. Raghav et al. [43] demonstrated no significant impact on survival and benefit of endocrine therapy in low $\mathrm{ER} / \mathrm{PR}+$ cases, which was similar to triple negative tumors. In the NSABP B-14 clinical trial Baehner and coworkers reported greater benefit from tamoxifen in patients with higher ER expression [44]. We analyzed our cases based on ER/PR expression and found that in the low $\mathrm{PR}+$ group of 48 (29\%) tumors, OS was significantly lower in ColX $\alpha 1$ high tumors. ColX $\alpha 1$ expression was associated with poor outcome in low PR+ tumors but not in high PR tumors. Like PR, other tumor stromal factors and tumor
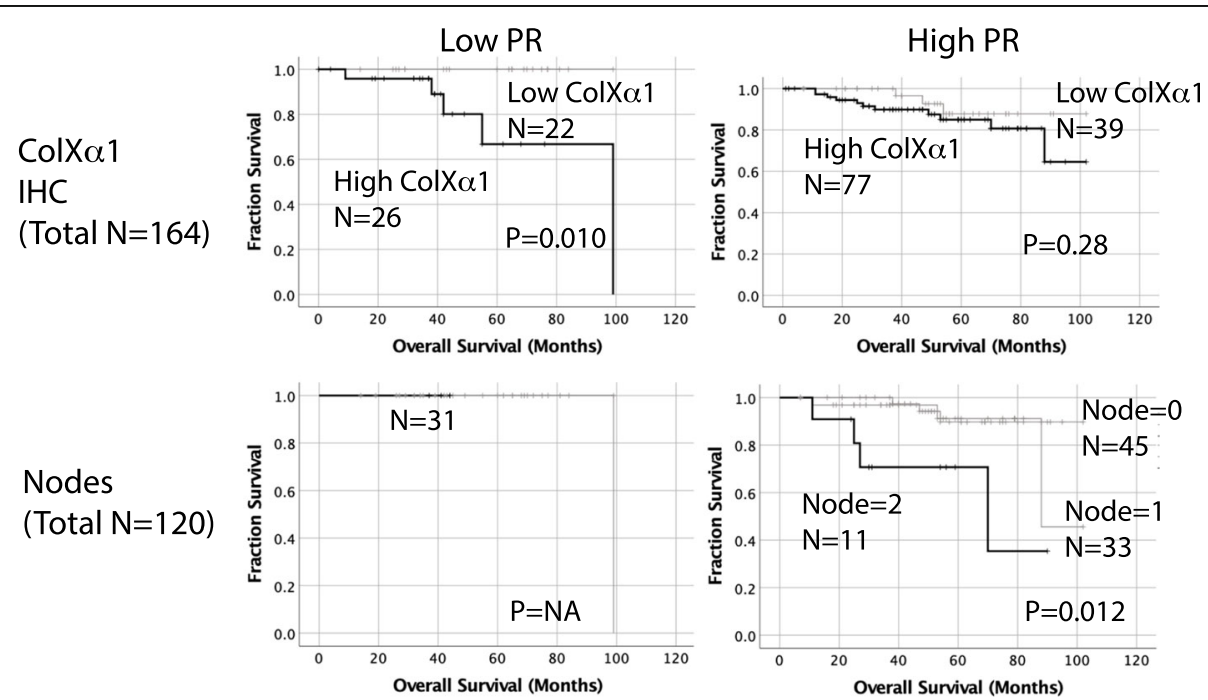

Fig. 3 Interaction and Impact of PR status, ColXa1 expression and number of metastatic nodes on survival. ColXa1 expression levels stratify only low PR+ tumors into prognostic groups (top left). Presence of lymph node metastasis was not a risk factor for patients with low PR levels but was a risk factor for patients with high PR levels. Nodes were grouped as follows: Node score =0, 0 nodes, Node score $=1,1-3$ nodes, Node score $=2,>3$ nodes 
microenvironment variables, such as ColX $\alpha 1$ have a potential to predict the treatment response and outcome.

\section{Conclusion}

Our findings indicate that extent of ColX $\alpha 1$ expression in the ER+/HER2+ breast tumor stroma is prognostic. Increased ColX $\alpha 1$ expression is associated with shorter OS and DFS in both neoadjuvant as well as adjuvant settings. Further molecular or proteomic studies are needed to refine the definition of ColX $\alpha 1$ positive/overexpressing tumors. The relationship of TILs and ColXolis intriguing, which needs further investigation, including correlation with imaging studies.

\section{Additional file}

Additional file 1: Table S1. Raw data for all cases in this study All data generated or analyzed during this study were included and de-identified (XLSX $26 \mathrm{~kb})$

\section{Abbreviations}

ColXa1: Type X collagen a1; DFS: Disease free survival; ER: Estrogen receptor; HER2: Human epidermal growth factor receptor 2;

IHC: Immunohistochemistry; OS: Overall survival; TILs: Tumor infiltrating lymphocytes

\section{Acknowledgments}

We thank Tara Szymanski from Breast Cancer Center assist in data collection. We thank the support from Dr. Douglas Anthony (Pathology-in-Chief of Lifespan Medical Center).

\section{Authors contributions}

YW and MBR conceived and designed the study, and prepared the manuscript. ASB performed the statistical analyses. CLZ, KS and YW collected and reviewed all the cases in the study including morphological and immunohistochemical evaluation. DY and AS performed the molecular experiments and related data analysis. MAF and TAG participated in design and data analysis. YW, CLZ and KS wrote the manuscript. SL, MAF, TAG and MBR reviewed the data and participated in revising the manuscript. All authors read and approved of the final manuscript.

\section{Funding}

This study was supported by the Molecular Pathology Core of the COBRE Center for Cancer Research Development funded by the National Institute of General Medical Sciences of the National Institutes of Health under Award Number P20GM103421 for histological tissue processing and immunohistochemistry analysis.

\section{Availability of data and materials}

All clinical pathological relevant information is summarized in Table 1 and raw data of all case was provided in Additional file 1: Table S1.

\section{Ethics approval and consent to participate}

This study was approved by the ethics committees of Lifespan Medical Center (467617-9) and Women Infants Hospital (797108-3). The need for consent was waived by the IRB.

\section{Consent for publication}

Not applicable.

\section{Competing interests}

Y.W, A. S. B and M. B. R. declare that a patent application has been approved 7/2017 titled as COLLAGENS AS MARKERS FOR BREAST CANCER TREATMENT to ASB, YW and MBR of Rhode Island Hospital, A Lifespan-Partner
(Application No. 15/187,279). No potential conflicts of interest were disclosed by other authors.

\section{Author details}

'Department of Pathology and Laboratory Medicine, Rhode Island Hospital and Lifespan Medical Center, Warren Alpert Medical School of Brown University, 593 Eddy St, APC 12, Providence, RI 02903, USA. ${ }^{2}$ Department of Pathology and Laboratory Medicine, Women and Infant Hospital, Warren Alpert Medical School of Brown University, Providence, RI 02903, USA. ${ }^{3}$ Department of Surgery, Rhode Island Hospital and Lifespan Medical Center, Warren Alpert Medical School of Brown University, Providence, USA. ${ }^{4}$ Department of Medicine, Rhode Island Hospital and Lifespan Medical Center, Warren Alpert Medical School of Brown University, Providence, USA.

Received: 8 January 2019 Accepted: 4 September 2019

Published online: 01 November 2019

\section{References}

1. Nasrazadani A, Thomas RA, Oesterreich S, Lee AV. Precision medicine in hormone receptor-positive breast Cancer. Front Oncol. 2018;8:144.

2. Slamon D, Eiermann W, Robert N, et al. Breast Cancer international research G. adjuvant trastuzumab in HER2-positive breast cancer. N Engl J Med. 2011; 365:1273-83.

3. Sorlie T, Tibshirani R, Parker J, et al. Repeated observation of breast tumor subtypes in independent gene expression data sets. Proc Natl Acad Sci U S A. 2003;100:8418-23.

4. Marchio C, Natrajan R, Shiu KK, et al. The genomic profile of HER2-amplified breast cancers: the influence of ER status. J Pathol. 2008;216(4):399-407.

5. $\mathrm{Ng}$ CK, Schultheis AM, Bidard FC, et al. Breast cancer genomics from microarrays to massively parallel sequencing: Paradigms and new insights. J Natl Cancer Inst. 2015;107(5):djv015.

6. Wirapati $\mathrm{P}$, Sotiriou $\mathrm{C}$, Kunkel $\mathrm{S}$, et al. Meta-analysis of gene expression profiles in breast cancer: toward a unified understanding of breast cancer subtyping and prognosis signatures. Breast Cancer Res. 2008;10:R65.

7. Gianni L, Eiermann W, Semiglazov V, et al. Neoadjuvant and adjuvant trastuzumab in patients with HER2-positive locally advanced breast cancer (NOAH): follow-up of a randomised controlled superiority trial with a parallel HER2-negative cohort. Lancet Oncol. 2014;15:640-7.

8. Gianni L, Eiermann W, Semiglazov V, et al. Neoadjuvant chemotherapy with trastuzumab followed by adjuvant trastuzumab versus neoadjuvant chemotherapy alone, in patients with HER2-positive locally advanced breast cancer (the NOAH trial): a randomised controlled superiority trial with a parallel HER2-negative cohort. Lancet. 2010;375:377-84.

9. Di Modica M, Tagliabue E, Triulzi T. Predicting the efficacy of HER2-targeted therapies: a look at the host. Dis Markers. 2017;2017:7849108.

10. Brodsky AS, Xiong J, Yang D, et al. Identification of stromal ColXalpha1 and tumor-infiltrating lymphocytes as putative predictive markers of neoadjuvant therapy in estrogen receptor-positive/HER2-positive breast cancer. BMC Cancer. 2016;16:274.

11. Bonnans C, Chou J, Werb Z. Remodelling the extracellular matrix in development and disease. Nat Rev Mol Cell Biol. 2014;15(12):786-801.

12. Kota J, Hancock J, Kwon J, Korc M. Pancreatic cancer: stroma and its current and emerging targeted therapies. Cancer Lett. 2017;391:38-49.

13. Gascard P, Tlsty TD. Carcinoma-associated fibroblasts: orchestrating the composition of malignancy. Genes Dev. 2016;30:1002-19.

14. Pickup MW, Laklai $\mathrm{H}$, et al. Stromally derived lysyl oxidase promotes metastasis of transforming growth factor-beta-deficient mouse mammary carcinomas. Cancer Res. 2013;73:5336-46.

15. Denkert C, Loibl S, Noske A, et al. Tumor-associated lymphocytes as an independent predictor of response to neoadjuvant chemotherapy in breast cancer. J Clin Oncol. 2010;28:105-13.

16. Hammond ME, Hayes DF, Dowsett M, et al. American Society of Clinical Oncology/College of American Pathologists guideline recommendations for immunohistochemical testing of estrogen and progesterone receptors in breast cancer. Archives of pathology and laboratory medicine. 2010;134(6): 907-22.

17. Wolff $\mathrm{AC}, \mathrm{Hammond} \mathrm{MEH}$, Allison $\mathrm{KH}$, et al. Human epidermal growth factor receptor 2 testing in breast Cancer: American Society of Clinical Oncology/ College of American Pathologists Clinical Practice Guideline Focused Update. J Clin Oncol. 2018;36(20):2105. 
18. Salgado R, Denkert C, Demaria S, et al, International TWG. The evaluation of tumor-infiltrating lymphocytes (TILs) in breast cancer: recommendations by an international TILs working group. Ann Oncol. 2015;26:259-71.

19. Broad Institute TCGA Genome Data Analysis Center. Analysis-ready standardized TCGA data from broad GDAC firehose stddata_2015_06_01 run. Dataset: Broad Institute of MIT and Harvard; 2015.

20. Carey LA, Perou CM, Livasy CA, et al. Race, breast cancer subtypes, and survival in the Carolina breast Cancer study. JAMA. 2006;295(21):2492-502.

21. Onitilo AA, Engel JM, Greenlee RT, Mukesh BN. Breast cancer subtypes based on ER/PR and Her2 expression: comparison of clinicopathologic features and survival. Clin Med Res. 2009;7(1-2):4-13.

22. Goldhirsch A, Winer EP, Coates AS, et al. Personalizing the treatment of women with early breast cancer: highlights of the St Gallen international expert consensus on the primary therapy of early breast Cancer 2013. Ann Oncol. 2013;24(9):2206-23.

23. McGuire A, Kalinina O, Holian E, Curran C, Malone CA, McLaughlin R, et al. Differential impact of hormone receptor status on survival and recurrence for HER2 receptor-positive breast cancers treated with Trastuzumab. Breast Cancer Res Treat. 2017:164:221-9.

24. Kennecke $H$, Yerushalmi R, Woods R, et al. Metastatic behavior of breast cancer subtypes. J Clin Oncol. 2010;28(20):3271-7.

25. Vaz-Luis I, Ottesen RA, Hughes ME, et al. Impact of hormone receptor status on patterns of recurrence and clinical outcomes among patients with human epidermal growth factor-2-positive breast cancer in the National Comprehensive Cancer Network: a prospective cohort study. Breast Cancer Res. 2012;14(5):R129.

26. Ribelles N, Perez-Villa L, Jerez JM, et al. Pattern of recurrence of early breast cancer is different according to intrinsic subtype and proliferation index. Breast Cancer Res. 2013;15(5):R98

27. Lowery AJ, Kell MR, Glynn RW, et al. Locoregional recurrence after breast cancer surgery: a systematic review by receptor phenotype. Breast Cancer Res Treat. 2011;133(3):831-41.

28. Osborne CK, Bardou V, Hopp TA, et al. Role of the estrogen receptor coactivator AIB1 (SRC-3) and HER-2/neu in tamoxifen resistance in breast cancer. J Natl Cancer Inst. 2003;95(5):353-61.

29. De Laurentiis M, Arpino G, Massarelli E, et al. A meta-analysis on the interaction between HER-2 expression and response to endocrine treatment in advanced breast cancer. Clin Cancer Res. 2005;11(13):4741-8.

30. Shou J, Massarweh S, Osborne CK, et al. Mechanisms of tamoxifen resistance: increased estrogen receptor-HER2/neu cross-talk in ER/HER2positive breast cancer. J Natl Cancer Inst. 2004;96(12):926-35.

31. Ioachim E, Charchanti A, Briasoulis E, et al. Immunohistochemical expression of extracellular matrix components tenascin, fibronectin, collagen type IV and laminin in breast cancer: their prognostic value and role in tumour invasion and progression. Eur J Cancer. 2002;38:2362-70.

32. Conklin MW, Keely PJ. Why the stroma matters in breast cancer: insights into breast cancer patient outcomes through the examination of stromal biomarkers. Cell Adhes Migr. 2012;6(3):249-60.

33. Aoudjit F, Vuori K. Integrin signaling inhibits paclitaxel-induced apoptosis in breast cancer cells. Oncogene. 2001;20(36):4995-5004.

34. Jobling R, D'Souza R, Baker N, et al. The collagenopathies: review of clinical phenotypes and molecular correlations. Curr Rheumatol Rep. 2014;16(1):394.

35. Finak G, Bertos N, Pepin F, et al. Stromal gene expression predicts clinical outcome in breast cancer. Nat Med. 2008;14:518-27.

36. Ma XJ, Dahiya S, Richardson E, Erlander M, Sgroi DC. (2009) Gene expression profiling of the tumor microenvironment during breast cancer progression. Breast Cancer Res 11(1):R7.Conklin MW, Eickhoff JC, Riching KM, et al (2011).

37. Aligned collagen is a prognostic signature for survival in human breast carcinoma. Am J Pathol 178(3):1221-1232

38. Marr MTn, Marr MTn, D \& apos, Alessio JA (2007) IRES-mediated functional coupling of transcription and translation amplifies insulin receptor feedback. Genes Dev 21(2):175-183.

39. Acerbi I, Cassereau L, Dean I, et al. Human breast cancer invasion and aggression correlates with ECM stiffening and immune cell infiltration. Integr Biol (Camb). 2015;7(10):1120-34.

40. Chapman KB, Prendes MJ, Sternberg H, et al. COL10A1 expression is elevated in diverse solid tumor types and is associated with tumor vasculature. Future Oncol. 2012;8(8):1031-40.

41. Huang H, Li T, Ye G, Zhao L, Zhang Z, Mo D, et al. High expression of COL10A1 is associated with poor prognosis in colorectal cancer. OncoTargets and Therapy. 2018;11:1571-81.
42. Wang Y, Lu S, Xiong J, Singh K, Hui Y, Zhao C, et al. ColXa1 is a stromal component that Colocalizes with elastin in the breast tumor extracellular matrix. J Pathol Clin Res. 2018;(Sep 12).

43. Raghav KPS, Hernandez-Aya LF, Lei X, et al. Impact of low estrogen/ progesterone receptor expression on survival outcomes in breast cancers previously classified as triple negative breast cancers. Cancer. 2012;118(6): 1498-506.

44. Baehner FL, Watson D, Shak S, et al (2006) Quantitative RT-PCR analysis of ER and PR by Oncotype DX indicates distinct and different associations with prognosis and prediction of tamoxifen benefit [abstract 45]. In 29th Annual San Antonio Breast Cancer Symposium 2006.

\section{Publisher's Note}

Springer Nature remains neutral with regard to jurisdictional claims in published maps and institutional affiliations.
Ready to submit your research? Choose BMC and benefit from:

- fast, convenient online submission

- thorough peer review by experienced researchers in your field

- rapid publication on acceptance

- support for research data, including large and complex data types

- gold Open Access which fosters wider collaboration and increased citations

- maximum visibility for your research: over $100 \mathrm{M}$ website views per year

At BMC, research is always in progress.

Learn more biomedcentral.com/submissions 